Annuaire suisse de politique de développement

18 | 1999

La Suisse et l'action humanitaire

\title{
1. Relations financières internationales
}

\section{Bruno Gurtner}

\section{OpenEdition}

Journals

Édition électronique

URL : http://journals.openedition.org/aspd/679

DOI : 10.4000/aspd.679

ISSN : 1663-9669

\section{Éditeur}

Institut de hautes études internationales et du développement

\section{Édition imprimée}

Date de publication : 1 janvier 1999

Pagination : $99-125$

ISSN : 1660-5934

\section{Référence électronique}

Bruno Gurtner, «1. Relations financières internationales », Annuaire suisse de politique de

développement [En ligne], 18| 1999, mis en ligne le 14 juillet 2012, consulté le 07 septembre 2020 URL : http://journals.openedition.org/aspd/679 ; DOI : https://doi.org/10.4000/aspd.679 


\section{RELATIONS FINANCIÈRES INTERNATIONALES*}

- 'ÉLÉMENT marquant de la turbulente année 1998 a été la crise financière. Devenue manifeste depuis le milieu de 1997 chez quelques "tigres» asiatiques, elle a pris de l'ampleur pour s'étendre à la Russie et à d'autres nations de la CEI et d'Europe orientale confrontées à de gigantesques problèmes intérieurs. L'Afrique du Sud et quelques pays d'Amérique latine ont également été touchés, tandis que la crise économique et financière du Japon faisait obstacle à des interventions efficaces. Les pays industrialisés du Nord craignent des répercussions fâcheuses sur la marche de leurs affaires. De grandes institutions financières ont subi des pertes massives. Cette crise a provoqué une transformation profonde des flux financiers, caractérisée par une forte diminution des financements publics et privés dans les pays en développement et en transition. Les institutions multilatérales ont dû multiplier les crédits à court terme. Dans ce contexte, la stratégie adoptée par le Fonds monétaire international (FMI) et le rôle que joue cette organisation ont fait l'objet de virulentes critiques.

\subsection{FLUX FINANCIERS VERS LES PAYS EN DÉVELOPPEMENT ET EN TRANSITION}

Après plusieurs années de croissance des flux financiers à destination des pays en développement et en transition, on assiste à un renversement de tendance. L'Organisation de coopération et de développement économiques (OCDE) indique que ces flux ont passé de 368 milliards de dollars en 1996 à 272 milliards en $1997^{1}$ (tableau $\left.n^{\circ} 1\right)$.

Le volume aussi bien que les types de flux financiers varient considérablement d'une région à l'autre. C'est ainsi que l'Afrique subsaharienne a reçu, en 1997, 27 dollars par habitant sous forme d'aide au développement et seulement 3 dollars d'investissements directs en provenance de l'étranger. Les chiffres correspondants ont été de 13 dollars (aide au développement) et de 62 dollars (investissements directs) pour les pays d'Amérique latine et des Antilles.

Contrairement à l'OCDE, le rapport Global Development Finance 1998 de la Banque mondiale annonce que les afflux nets ont encore progressé de 18,7 milliards de dollars en 1997, atteignant ainsi 300,3 milliards de dollars². Mais la Banque mondiale s'attend également - tout comme le FMI, la CNUCED, la Banque des règlements internationaux à Bâle (BRI) et l'Institute of International Finance (IIF) à Washington (centre d'études des grandes banques commerciales privées) - à un ralentissement des flux de capitaux pour 1998.

* Par Bruno Gurtner, économiste, Communauté de travail, Berne.

1. OCDE, L'aide et les apports de capitaux privés ont diminué en 1997, communiqué de presse, Paris, 18.6.98. Les chiffres présentés ici diffèrent par rapport à la statistique de l'année précédente en raison de changements dans la liste des pays bénéficiaires et dans celle des pays donateurs.

2. World Bank, Global Development Finance 1998, Washington, 1998. Pour les divergences statistiques entre OCDE, Banque mondiale, FMI, CNUCED et BRI, cf. ASTM 1997, pp. 13-14. 
Tableau $n^{\circ}$ 1: Flux de capitaux nets à destination des pays en développement et en transition (en milliards de dollars aux taux actuels)

\begin{tabular}{lrrr}
\hline & $\mathbf{1 9 9 5}$ & $\mathbf{1 9 9 6}$ & $\mathbf{1 9 9 7}^{\circ}$ \\
\hline I. Financement public du développement (APD) & $\mathbf{8 9 . 3}$ & $\mathbf{7 8 . 1}$ & $\mathbf{7 0 . 0}$ \\
\hline 1. Aide publique au développement (APD) & 59.8 & 57.9 & 49.0 \\
\hline dont: versements bilatéraux & 40.6 & 39.1 & 31.0 \\
\hline versements multilatéraux & 19.2 & 18.9 & 18.0 \\
\hline 2. Autres FPD & 21.1 & 14.5 & 14.0 \\
\hline dont: versements bilatéraux & 14.0 & 5.8 & 6.0 \\
\hline$\quad$ versements multilatéraux & 7.1 & 8.7 & 8.0 \\
\hline II. Crédits à l'exportation & $\mathbf{5 . 6}$ & $\mathbf{4 . 0}$ & $\mathbf{- 4 . 4}$ \\
\hline III. Apports privés & $\mathbf{1 6 8 . 3}$ & $\mathbf{2 8 6 . 3}$ & $\mathbf{2 0 6 . 2}$ \\
\hline 1. Investissements directs & 52.3 & 64.3 & 75.0 \\
\hline 2. Prêts bancaires internationaux* & 76.9 & 86.0 & 12.0 \\
\hline 3. Prêts obligataires & 30.0 & 96.6 & 83.2 \\
\hline 4. Autres apports privés** & 3.1 & 33.8 & 30.0 \\
\hline 5. Dons des ONG & 6.0 & 5.6 & 6.0 \\
\hline Total des apports nets de ressources & $\mathbf{2 6 3 . 2}$ & $\mathbf{3 6 8 . 4}$ & $\mathbf{2 7 1 . 8}$ \\
\hline
\end{tabular}

Source: OCDE, L'aide et les apports de capitaux privés ont diminué en 1997

* A l'exclusion des prêts obligataires consentis par les banques (rubrique III.3) et des crédits financiers garantis (sous II).

** Certains pays du CAD font des rapports incomplets; Japon inclus depuis 1996.

${ }^{\mathrm{P}}$ Chiffres provisoires.

\section{$\square$ Financement public du développement: en perte de vitesse}

Selon l'OCDE, l'aide publique au développement (APD) octroyée par ses membres se chiffrait en 1997 à 0,22\% de leur produit national brut (PNB), ce qui est la valeur la plus basse jamais enregistrée. Canada mis à part, ce recul est dû pour l'essentiel aux grands pays du Groupe des sept (G7), dont la part du PNB consacrée à l'APD est tombée à $0,19 \%$. Cette part se situe à $0,45 \%$ pour les autres pays de l'OCDE. L'APD a progressé en valeur réelle - compte tenu de l'inflation et des fluctuations monétaires - chez 11 des 14 pays qui ne font pas partie du G7. L'APD de la Suisse atteint $0,32 \%$ de son PNB alors que ce taux était de $0,34 \%$ l'année précédente, ce qui représente un recul de $4,2 \%$ en valeur réelle.

$\square$ ASTM 1999, chap. 8, partie 8.1.

La Banque mondiale ${ }^{3}$ relève que la compression de leurs budgets de développement conduit les pays donateurs à rendre leur travail de coopération encore plus efficace. Les fonds disponibles vont de plus en plus aux pays qui s'engagent dans une politique de réformes, et les agences officielles prennent des mesures propres à stimuler des afflux durables de capitaux privés. Le rapport 1997 de l'OCDE s'exprime également sur ces questions ${ }^{4}$. Le FMI, de son côté, fait remarquer ${ }^{5}$ que les pays en développement démunis restent tributaires d'une aide publique à taux préférentiels.

Le volume considérable de crédits transitoires qu'il a fallu octroyer aux pays en crise vers la fin de 1997 a relancé le mouvement des crédits à taux non préférentiels qui reculaient ces dernières années.

3. World Bank, Global Development Finance 1998, pp. 5 et 49sqq.

4. OCDE, Coopération pour le développement. Efforts et politiques des membres du Comité d'aide au développement. Rapport 1997, Paris, 1998.

5. OCDE, L'aide et les apports de capitaux privés... 


\section{$\square$ Flux financiers privés: fin de la croissance}

On assistait depuis 1990 à une augmentation persistante des flux de capitaux privés à destination des pays en développement et en transition; cette évolution a pris fin avec la crise financière qui a secoué le Sud-Est asiatique au milieu de 1997. L'OCDE a calculé pour l'ensemble de cette année-là un afflux de 206 milliards de dollars, soit 80 milliards de moins que l'année précédente (tableau n 1 ).

L'OCDE observe ${ }^{6}$ que l'afflux toujours croissant de capitaux privés vers l'Amérique latine ne compense pas le recul des crédits bancaires à destination des pays asiatiques. Les investissements directs à l'étranger continuent de progresser globalement, tandis que toutes les autres formes d'apports privés reculent - notablement pour certaines - par rapport à l'année précédente. Selon ces chiffres provisoires de l'OCDE, l'essentiel de ces mouvements de capitaux continue d'affluer vers les pays les plus dynamiques d'Asie, d'Europe et d'Amérique du Sud. Les capitaux privés allant au groupe des pays pauvres ont totalisé 22 milliards de dollars, essentiellement à destination de l'Inde et de la Chine. Les pays de l'Afrique subsaharienne n'ont obtenu ensemble que 2 milliards de dollars en investissements directs et crédits bancaires.

D'autres statistiques confirment ce changement de tendance. Selon des estimations de l'Institute of International Finance (IIF $)^{7}$, les investissements ont continué d'augmenter en 1997, mais les placements de portefeuille ont nettement régressé par rapport à 1996, et les crédits bancaires ont même eu un solde négatif en ce sens que les remboursements ont dépassé les nouveaux crédits.

La Banque des règlements internationaux (BRI) confirme que les afflux nets de capitaux internationaux - essentiellement sous forme de crédits bancaires à court terme - ont passé de pratiquement zéro en 1989 à un maximum de presque 170 milliards de dollars en 1996; cette croissance a ensuite cédé la place à un reflux de grande ampleur ${ }^{8}$. Les statistiques trimestrielles de la BRI mettent en chiffres ce dramatique renversement de tendance pour les cinq pays d'Asie les plus touchés: les prêts/crédits interbancaires et les prêts/crédits au secteur privé non bancaire ont encore augmenté de 22 milliards de dollars entre le deuxième trimestre 1996 et le troisième trimestre 1997, tandis que le mouvement s'est inversé pour chuter de 32 milliards durant le dernier trimestre 1997 et de 34 milliards pour le premier trimestre de 1998.

La Banque mondiale ${ }^{9}$ signale au contraire que les flux de capitaux privés ont atteint 256 milliards de dollars en 1997, dépassant ainsi les 247 milliards de l'année précédente. Mais s'il y a croissance, celle-ci est bien ralentie. Toutes les formes de flux financiers privés y ont contribué (cf. ASTM 1998, p. 138, encadré sur les flux financiers privés). Seuls les investissements de portefeuille n'ont pas atteint le niveau de l'année précédente, plafonnant à celui de 1994 et 1995. La Banque mondiale publie ses chiffres provisoires avant d'autres institutions, mais en se basant sur des statistiques plus anciennes (voir plus bas l'encadré «Statistiques de la BRI concernant l'activité bancaire internationale»). Elle souligne toutefois que les flux financiers privés ralentiront sans doute dans un

6. OCDE, L'aide et les apports de capitaux privés...

7. Citées par la NZZ, «Abrupt versiegende Kapitalströme im Jahre 1997», 30.1.98.

8. Banque des règlements internationaux (BRI), 68 rapport annuel, Bâle, 1998, p. 187.

9. World Bank, Global Development Finance 1998, Table 1. 
proche avenir - tendance qui devrait moins toucher les investissements directs que les investissements en portefeuille, les obligations et les crédits bancaires.

La Banque mondiale constate par ailleurs que le climat de volatilité et d'insécurité n'a pas empêché de nombreux pays en développement et en transition de participer plus activement au marché financier international, sous des formes parfois innovantes.

\section{$\square$ La crise financière}

L'actuelle crise financière a fait couler beaucoup d'encre. Institutions internationales, gouvernements, banques commerciales, médias, milieux scientifiques, ONG ont publié un nombre incalculable d'études et d'articles sur ses causes, sur le cours des événements, sur leur évolution probable, les mesures à prendre, sur la critique des institutions, etc. La récapitulation ci-après est par la force des choses limitée.

[C] ASTM 1998, pp. 139sqq. sur la crise financière dans les pays nouvellement industrialisés d'Asie.

La crise financière du Sud-Est asiatique a d'abord éclaté en Thaillande au mois de juillet 1997, pour s'étendre ensuite inexorablement à d'autres pays et régions du monde. A part les autres nations nouvellement industrialisées d'ExtrêmeOrient, elle a frappé la Chine, le Japon, la Russie ainsi que d'autres pays de la CEI et d'Europe orientale. La crise a enfin menacé des pays sud-américains (Brésil, Venezuela, Argentine) et l'Afrique du Sud. Sa propagation a d'ailleurs été facilitée par les problèmes internes de certains pays.

Il n'est pas certain, au dire de la $\mathrm{BRI}^{10}$, que le pire soit passé. Les répercussions économiques et sociales de la crise devront encore être analysées et il faudra voir dans quelle mesure les entreprises indigènes et leurs créanciers en ont été affectés. Le choc a été d'autant plus grand que l'Asie faisait figure de modèle d'avenir. Les commentateurs ont été de plus en plus nombreux à percevoir une importante crise structurelle au-delà des problèmes de trésorerie.

La BRI considère que la crise asiatique s'explique par l'économie intérieure des pays affectés: croissance excessive des crédits au secteur privé et plus particulièrement des dettes à court terme - d'où un gonflement démesuré des capitaux engagés, une surveillance insuffisante des systèmes bancaires et financiers, des valeurs surfaites et une rigidité des changes empêchant une politique monétaire plus flexible. L'appréciation du dollar à partir de 1995 et la chute des prix à l'exportation - pour les produits électroniques notamment - ont donné à ces pays la tentation de dévaluer pour se rendre compétitifs. Un manque de transparence n'a pas permis au marché international de discerner à temps et avec suffisamment de netteté les faiblesses qui se faisaient sentir dans le secteur financier. Par contre, l'inflation et les déficits publics ont gardé des proportions modestes.

Mais il serait faux, selon la BRI, d'en tirer la conclusion qu'une meilleure politique économique des pays considérés aurait suffi pour éviter toutes ces difficultés. Des déséquilibres économiques d'envergure planétaire sont pour beaucoup dans cette évolution asiatique et pourraient également se manifester sous d'autres formes. 
La BRI relève en particulier que la forte augmentation des capitaux disponibles à l'échelon mondial est due aux décalages conjoncturels étonnamment persistants entre les grandes nations industrialisées, ainsi qu'à une politique monétaire partout axée sur la stabilité assortie de faibles taux d'intérêt. Ces liquidités ont commencé par gonfler les flux financiers à destination des marchés émergents. Dans l'optique de la BRI, les investisseurs soucieux de maintenir le niveau de leurs rendements ont été tentés de négliger l'augmentation des risques.

Lorsque la crise financière a éclaté, les investisseurs ont essayé de retirer massivement leurs capitaux des marchés menacés (voir plus haut la partie «Flux financiers privés: fin de la croissance»). Ce reflux a considérablement aggravé la crise. Les investisseurs se sont réfugiés dans la qualité, de sorte que les placements de premier ordre ont dans l'ensemble évolué à la baisse durant l'année écoulée.

Mais cette crise a aussi conduit à accélérer les réformes du secteur financier qui étaient devenues indispensables à l'échelle mondiale. La BRI estime que macroéconomie et stabilité financière vont de pair, de sorte qu'il convient de renforcer le système bancaire et la finance. Il faut assurer d'une part un meilleur accès aux informations, raison pour laquelle toujours plus de pays adoptent les Special Data Dissemination Standards du Fonds monétaire international (FMI). C'est là un moyen de déceler plus tôt et plus sûrement les situations de crise. Elaborés sous l'égide de la BRI par le Comité de Bâle sur le contrôle bancaire en collaboration avec les autorités de surveillance des pays émergents, les Principes fondamentaux pour un contrôle bancaire efficace ont de leur côté une fonction préventive et devront être appliqués de manière systématique. Ces principes englobent entre autres une certaine normalisation et une transparence accrue de la comptabilité des banques. La communauté internationale, emmenée par le FMI, a enfin octroyé aux pays affectés des aides financières considérables afin de stabiliser cette situation de crise (voir plus bas la partie «Le Fonds monétaire international»). Des efforts ont par ailleurs été entrepris dans certains cas pour inciter les grandes banques à rééchelonner ou reconduire les dettes échues. La $\mathrm{BRI}$ considère que le secteur privé devrait se montrer dans une certaine mesure disposé à octroyer de nouveaux fonds aux débiteurs qui ont bénéficié dans le passé de crédits trop faciles (NZZ, 9.6.98).

En ce qui concerne les critiques parfois virulentes qu'ont suscité les interventions du FMI, la BRI fait remarquer que c'est la première crise depuis la Seconde Guerre mondiale où les principaux créanciers internationaux sont des banques et où les plus importants débiteurs appartiennent au secteur privé. Il n'existe donc pas de solution éprouvée pour venir à bout de la crise asiatique, et il faudra des années pour en tirer toutes les leçons.

\section{Statistiques de la BRI concernant l'activité bancaire internationale}

La BRI constate que ses statistiques des opérations bancaires internationales font l'objet d'un regain d'attention depuis que la crise asiatique a éclaté. Ses statistiques bancaires trimestrielles se réfèrent au lieu d'implantation des banques qui l'informent. Elles répertorient les créances et les dettes de banques commerciales domiciliées dans les 24 pays - 18 nations industrialisées et six autres places financières - dont la BRI reçoit des comptes rendus. Ces statistiques sont ventilées par types de débiteurs (banques et non-banques) et par monnaies de créances; elles indiquent ainsi le volume et la nature des flux financiers internationaux et transnationaux. 
Les statistiques bancaires semestrielles consolidées présentent les crédits bancaires accordés aux différents pays, ventilés par secteurs, échéances et nationalités des prêts bancaires internationaux. Ces statistiques répertorient les créances des banques de 18 pays à l'égard de pays en développement, de pays de l'Europe orientale et de pays qui ne fournissent pas de renseignements à la BRI.

La BRI s'efforce d'améliorer encore la qualité de ses informations et de les tenir à jour. Ses statistiques semestrielles révélaient quelque temps avant la crise de 1997 que la dette extérieure des pays asiatiques avait beaucoup augmenté. C'est ainsi que cette institution a mis en évidence dès 1994 la forte croissance des dettes à court terme de la Thaïlande.

Les statistiques de la BRI se basent sur des chiffres de créanciers; elles peuvent être considérées comme un éclairage différent et un complément aux statistiques de l'endettement publiées par la Banque mondiale, fondées, elles, sur les données des pays débiteurs. Les chiffres de la BRI sont plus récents du fait que le délai de publication est de cinq à six mois, alors que ceux des débiteurs de la Banque mondiale remontent à une ou deux années. Dans certains cas, le montant des dettes inventoriées par la BRI est plus élevé que celui de la Banque mondiale, ce qui permet peut-être de conclure que certains pays débiteurs ne déclarent pas entièrement leurs dettes.*

* BRI, Activité bancaire et financière internationale, Bâle, août 1998, pp. 33sqq.

\subsection{ENDETTEMENT EXTÉRIEUR}

L'initiative en faveur des pays pauvres très endettés (PPTE) est restée au centre des débats sur l'endettement, qui ont abordé la question de savoir comment traiter les arriérés et les dettes excédentaires des post-conflict countries ${ }^{11}$. Des mesures particulières doivent être prises en vue d'aider ces pays à se qualifier en tant que bénéficiaires de l'initiative PPTE.

Pour les autres pays, la discussion s'est concentrée sur leur intégration croissante dans les marchés financiers (privés) globaux, sur la forme qu'il convient de donner à cette intégration, sur les échéances et les risques. Cette évolution confère une importance croissante aux garanties sur les crédits d'exportation et les investissements. La Banque mondiale note que l'Indonésie mise à part, les pays du Sud-Est asiatique avaient en 1996 un ratio d'endettement (rapport entre la dette et les recettes d'exportation) inférieur de moitié à celui des nations latino-américaines lourdement endettées ${ }^{12}$. Mais le Club de Paris - où se restructurent les dettes officielles - n'a pas chômé pour autant. On y a essentiellement discuté de meilleures conditions pour le rééchelonnement de la dette des pays les plus pauvres, ainsi que le cas de la Russie. Les opérations d'aménagement des dettes commerciales ont continué sur leur lancée. Certains pays ont vu leurs dettes intérieures devenir un problème pesant. La Suisse a poursuivi, dans le domaine de l'endettement, la politique éprouvée qui lui vaut l'approbation de la communauté internationale.

\section{$\square$ Tendances}

Selon des informations provisoires de la Banque mondiale ${ }^{13}$, les dettes des pays en développement et en transition ont progressé de 76 milliards de dollars pour

11. Pays qui ont mis fin à un conflit (p. ex. pays de l'ex-Yougoslavie, Soudan, Rwanda, Burundi). La Banque mondiale doit préparer un document de travail sur ce sujet pour sa session du printemps 1999.

12. World Bank, Global Development Finance 1998, fig. 2.1, p. 33

13. World Bank, Global Development Finance 1998, pp. 160-161. 
atteindre 2171,4 milliards. Sur ce total, 1728,5 milliards sont des dettes à long terme (tableau $\mathrm{n}^{\circ} 2$ ). Quant à la répartition géographique, l'endettement augmente partout sauf dans les pays de l'Afrique subsaharienne, où il a quelque peu reculé. Les pays les plus pauvres lourdement endettés ont également vu leur passif se tasser légèrement.

Tableau n²: Endettement des pays en développement et en transition (en milliards de dollars)

\begin{tabular}{lrrrr}
\hline & $\mathbf{1 9 8 0}$ & $\mathbf{1 9 9 0}$ & $\mathbf{1 9 9 6}$ & $\mathbf{1 9 9 7}^{\mathbf{0}}$ \\
\hline Endettement à long terme & 445 & 1168 & 1650 & 1729 \\
\hline $\begin{array}{l}\text { - de source publique } \\
\text { (bilatéral et multilatéral) }\end{array}$ & 171 & 595 & 828 & 820 \\
\hline - de source privée & 207 & 513 & 569 & 609 \\
\hline Endettement à court terme & 147 & 241 & 385 & 381 \\
\hline Crédits du FMl & 12 & 35 & 60 & 62 \\
\hline Endettement cumulé & $\mathbf{6 0 3}$ & $\mathbf{1 4 4 4}$ & $\mathbf{2 0 9 5}$ & $\mathbf{2 1 7 1}$ \\
\hline
\end{tabular}

Tableau n³: Indices d'endettement 1996-1997

\begin{tabular}{lrrrr}
\hline $\begin{array}{l}\text { Catégories de pays } \\
\text { (en \%) }\end{array}$ & $\begin{array}{r}\text { d/ex* } \\
\mathbf{1 9 9 6}\end{array}$ & $\begin{array}{r}\text { d/ex } \\
\mathbf{1 9 9 7 p}\end{array}$ & $\begin{array}{r}\text { sd/ex** } \\
\mathbf{1 9 9 6}\end{array}$ & $\begin{array}{r}\mathbf{s d / e x} \\
\mathbf{1 9 9 7 p}\end{array}$ \\
\hline Tous les pays en développement & 137,4 & 134,3 & 17,2 & 16,7 \\
\hline - très endettés, bas revenus & 361,7 & & 15,3 & \\
\hline - très endettés, revenus moyens & 284,2 & & 36,0 & \\
\hline - moyennement endettés, bas revenus & 197,2 & & 22,9 & \\
\hline - moyenn. endettés, revenus moyens & 116,6 & & 21,2 & \\
\hline - Andere & 79,2 & & 8,0 & \\
\hline Régions & & & & \\
\hline - Afrique subsaharienne & 221,6 & 201,7 & 14,2 & 11,5 \\
\hline - Asie de l'Est et Pacifique & 101,8 & 102,6 & 13,0 & 11,7 \\
\hline - Asie du Sud & 193,6 & 179,8 & 22,0 & 21,0 \\
\hline - Europe et Asie centrale & 104,8 & 102,0 & 11,4 & 10,6 \\
\hline - Amérique latine et Caraïbes & 198,0 & 192,6 & 32,3 & 34,1 \\
\hline - Moyen-Orient et Afrique du Nord & 111,4 & 114,9 & 11,4 & 10,5 \\
\hline
\end{tabular}

Source des tableaux 2 et 3: World Bank, Global Development Finance 1998, Summary Tables pp. 159sqq

* ad/ex = rapport dette/exportations de biens et services.

** bsd/ex = rapport service de la dette/exportations de biens et services.

Données provisoires.

\section{$\square$ Initiative en faveur des PPTE}

Lancée en 1996, cette initiative multilatérale en faveur des pays pauvres lourdement endettés a pour but de ramener leur dette à des proportions tolérables tout en stimulant des réformes économiques et sociales qui devraient aider ces pays à sortir de l'ornière. Les pays classés PPTE sont au nombre de 41 (33 africains, 4 asiatiques et 4 latino-américains). Leur part du total des dettes répertoriées se chiffre à $12 \%$. L'endettement est considéré comme viable lorsqu'il ne dépasse pas $250 \%$ des recettes d'exportation ou si le service de la dette ne représente pas plus de $25 \%$ de ces recettes. Le calcul englobe toutes les catégories de créanciers. Le pays qui entend se qualifier doit avoir mené à bien un programme d'ajustement structurel. La décision de le faire bénéficier de l'initiative sera prise trois ans après le début de ce programme (point de décision), et des mesures de désendettement supplémentaires seront prises après une seconde période de trois ans consacrée à l'achèvement du programme (point d'achèvement). 
Depuis avril 1997, sept pays se sont qualifiés en parvenant au «point de décision»: l'Ouganda, la Bolivie, le Burkina-Faso, la Guyane, la Côte-d'Ivoire, le Mozambique et le Mali. Deux seulement avaient atteint le point d'achèvement à fin 1998 (tableau n4). Le Bénin ne s'est pas qualifié en raison d'un endettement insuffisant.

ASTM 1998, pp. 147sqq sur l'initiative en faveur des PPTE.

Tableau n4: Opérations de désendettement relevant de l'initiative en faveur des PPTE

\begin{tabular}{lcccc}
\hline Pays & Décision & Achèvement & $\begin{array}{c}\text { Désendettement } \\
\text { en mio. de \$ }\end{array}$ & $\begin{array}{c}\text { Désendettement } \\
\text { en \% de la dette }\end{array}$ \\
\hline Ouganda & avril 97 & avril 98 & 650 & 20 \\
\hline Bolivie & sept. 97 & sept. 98 & 760 & 13 \\
\hline Burkina Faso & sept. 97 & avril 2000 & 200 & 14 \\
\hline Guyane & déc. 97 & début 99 & 500 & 25 \\
\hline Côte d'lvoire & mars 98 & mars 2001 & 800 & 6 \\
\hline Mozambique & avril 98 & juin 99 & 2900 & 57 \\
\hline Mali & sept. 98 & déc. 99 & 250 & 10 \\
\hline Total & & & $\mathbf{6 0 6 0}$ & \\
\hline
\end{tabular}

Source: IMF, Debt Initiative for the Heavily Indebted Poor Countries, Fact Sheet, September 1998

Le principe des trois ans nécessaires pour atteindre les points de décision et d'achèvement a été assoupli (délais raccourcis) pour les sept pays ci-dessus. D'autres bénéficiaires potentiels - notamment Ethiopie, Guinée, Guinée-Bissau, Mauritanie, Niger, Sénégal, Sierra Leone, Togo, Tchad et Vietnam - sont actuellement à l'étude, mais il ne faut pas s'attendre à ce que tous ces pays remplissent les conditions requises pour se qualifier.

Le FMI et la Banque mondiale ont dressé un Progress Report ${ }^{14}$ peu avant la session de printemps et leur session annuelle d'octobre 1998. Les directoires du FMI et de la Banque mondiale ont prolongé le délai d'entrée jusqu'à fin 2000. Les post-conflict countries peuvent également se qualifier s'ils prennent des mesures qui leur permettent de satisfaire aux critères de l'initiative PPTE. Cette extension grossit le coût global à prévoir, qui est actuellement estimé à 9,7 milliards de dollars.

Le financement n'est pas encore réglé de manière définitive. La Banque mondiale a transféré jusqu'ici 750 millions de dollars en gains courants sur le fonds fiduciaire PPTE. En juillet, le conseil d'administration de la Banque mondiale a recommandé de verser sur le même fonds encore 100 millions provenant du bénéfice net de 1998. Quant à la Suisse, le Conseil fédéral a débloqué le 18 août 1998 la seconde tranche de 20 millions de francs au titre de la contribution helvétique au fonds fiduciaire PPTE de la Banque mondiale, contribution qui se monte ainsi à 40 millions de francs. Par ailleurs, l'Office fédéral des affaires économiques extérieures (OFAEE) est le principal initiateur d'un programme de promotion de l'autonomie locale (capacity-building programme). La Suisse s'est associée au Danemark, à l'Autriche et à la Suède pour financer - d'entente avec le FMI et la Banque mondiale - les moyens techniques que requiert l'élaboration de stratégies nationales de désendettement (voir plus bas la partie «Programme suisse de désendettement»). 
Le FMI, pour sa part, estime disposer des fonds nécessaires pour sa contribution au financement courant de la Facilité d'ajustement structurel renforcée (FASR), mais ce financement n'est pas encore tout à fait réglé à moyen terme. Un autre rapport, consacré au financement par des apports bilatéraux et, le cas échéant, par des réserves d'or, devrait sortir vers la fin de 1998. La Suisse participe à hauteur de 45 millions de francs au renouvellement du fonds fiduciaire de la FASR, qui servira à alimenter l'initiative en faveur des PPTE.

Le FMI et la Banque mondiale ont essuyé de vives critiques concernant la manière dont cette initiative a été conçue et réalisée, raison pour laquelle les deux institutions ont invité des gouvernements et des ONG à un séminaire qui s'est tenu en février à Paris. La Suisse y était représentée par l'OFAEE et la Communauté de travail des œuvres d'entraide. Les critiques de l'ONG Eurodad portaient sur la définition de la «viabilité de la dette extérieure», la prise en compte de critères fiscaux, la lenteur des opérations, le manque d'assistance jusqu'au point d'achèvement. Les évaluations devraient en outre prendre en considération des indicateurs de pauvreté, et la preuve devrait être apportée que les allégements de dette conduisent à un développement social ${ }^{15}$.

\section{$\square$ Réaménagement de dettes publiques}

La plupart des rééchelonnements de dettes publiques bilatérales sont négociés dans le cadre du Club de Paris. Il s'agit là de crédits octroyés par des gouvernements à d'autres gouvernements, auxquels s'ajoutent des crédits d'exportation accordés aux conditions du marché et qui ont été repris par des institutions publiques au titre de la Garantie contre les risques à l'exportation (dans le cas de non-paiement aux créanciers privés). Le volume de ces garanties officielles a beaucoup augmenté au cours des années 90, d'une part en raison de la politique agressive de nombreux pays en matière de promotion des exportations, d'autre part du fait que le financement international du développement a changé de nature, comme le souligne la Banque mondiale ${ }^{16}$. En particulier, l'ampleur prise par le financement direct de projets d'infrastructure a accru les engagements des institutions nationales de garantie contre les risques à l'exportation. Ces organismes ont harmonisé depuis quelques années leur politique de crédit d'exportation sous la forme du «consensus de l'OCDE». Les efforts des ONG pour y inclure des critères écologiques et sociaux n'ont pas encore abouti ${ }^{17}$. On en est resté à une vague déclaration d'intention politique ${ }^{18}$. En octobre 1998, l'OCDE a invité les représentants d'ONG internationales à des premiers pourparlers.

Les difficultés financières croissantes des pays les plus pauvres ont conduit à assouplir les conditions de réaménagement de leur dette. Après le sommet du G7 de 1994 à Naples, les pays créanciers se sont entendus pour octroyer au besoin des conditions de faveur sur deux tiers de la dette à réaménager; le cas échéant, les opérations de rééchelonnement portent non seulement sur les échéances, mais aussi sur l'encours de la dette. Dans le cadre de l'initiative en faveur des PPTE,

15. World Bank, HIPC Debt Initiative: Taking Stock and Looking Ahead, Conference Documents, The World Bank, February 3, 1998, Paris.

16. World Bank, Global Development Finance, p. 58.

17. Lettre non publiée de Environmental Defense Fund (Etats-Unis), Eurodad (Bruxelles) et Communauté de travail des œuvres d'entraide du 16.12.97.

18. Info@Bawi n², Berne, 1998. 
les créanciers ont résolu d'accorder selon les cas des réductions pouvant atteindre $80 \%$ de la dette à rééchelonner.

ASTM 1998, pp. 151-152 sur le réaménagement de dettes dans le cadre du Club de Paris.

Les accords de réaménagement multilatéraux $d u$ Club de Paris ont été au nombre de sept en 1997, pour un montant total de 6,3 milliards de dollars ${ }^{19}$. La réduction accordée a été de $67 \%$ pour l'Ethiopie, Madagascar, la Tanzanie et la République du Yémen, et de $50 \%$ pour la Cameroun et la Guinée. Tous ces pays appartiennent à la catégorie des PPTE. Un rééchelonnement a également eu lieu en 1997 pour la Jordanie, mais pas à des conditions de faveur du fait que celleci se classe dans les pays à revenus moyens. La Suisse contribue à ces opérations en faveur de quatre pays pour un montant global de 35,9 millions de francs.

En 1998, le Club de Paris avait pour 7,3 milliards de dollars de créances à l'égard des huit pays suivants: Côte-d'Ivoire, Sénégal, Rwanda, Albanie, Indonésie, République centrafricaine, Bosnie-Herzégovine et Bolivie.

Les réaménagements bilatéraux de la Suisse conclus en 1997 totalisent 425 millions de francs ${ }^{20}$ et ont été passés avec cinq pays, à savoir la Guinée Conakry (deux accords), le Cameroun, Madagascar, la Jordanie et la Russie. Au total, 383,1 millions de francs rééchelonnés dans le contexte bilatéral portaient sur des créances issues de précédentes conversions de dettes, qui ont ainsi fait l'objet d'une nouvelle consolidation. Il n'a été procédé à aucun rééchelonnement bilatéral en 1998.

\section{$\square$ Dettes commerciales}

En 1997, neuf accords de réaménagement ont été passés entre les pays débiteurs et leurs banques créancières - pour 19,1 milliards de dollars en dettes courantes et 6,9 milliards d'arriérés ${ }^{21}$. Parmi les pays à bas revenus, le Togo a racheté pour 46,1 millions de dollars de dettes au taux de 12,5\% avec l'aide du Fonds de réduction de la dette (Debt Reduction Facility) de l'AID (Association internationale de développement). La Côte-d'Ivoire (6,5 milliards de dollars) et le Vietnam (0,8 milliard) ont restructuré leurs dettes dans le cadre du Plan Brady. La BosnieHerzégovine a conclu avec le Club de Londres des banques commerciales un accord de rééchelonnement portant sur 1,3 milliard de dollars. Parmi les pays à revenus moyens, l'Argentine, le Brésil, l'Equateur, Panama et le Venezuela ont remplacé des obligations Brady par des obligations non garanties. La Russie, enfin, a passé en octobre 1997, avec les banques commerciales, un protocole de réaménagement portant sur 33 milliards de dollars.

\section{$\square$ Programme suisse de désendettement}

Les mesures de désendettement prises par la Suisse sont de quatre types:

- mesures bilatérales;

- rachat de dettes commerciales;

๑ mesures multilatérales;

- mesures complémentaires, dont l'assistance technique.

19. World Bank, Global Development Finance 1998, p. 82; Rapport GRE 97, Bureau GRE/OFAEE, Zurich/Berne, juin 1998, p. 19.

20. «Rapport sur la politique économique extérieure 1997», Feuille fédérale, 10.3.98, p. 605.

21. World Bank, Global Development Finance 1998, pp. 83-84. 
Des 500 millions de francs (400 millions du «fonds d'anniversaire» et 100 millions du crédit de programme destiné à des mesures de politique économique et commerciale) consacrés à des opérations de désendettement, 390 millions avaient été engagés à fin 1998; il reste donc 110 millions de francs à attribuer.

La Suisse a racheté depuis 1993 des créances bilatérales de 28 pays pauvres lourdement endettés; 19 pays ont ainsi bénéficié de remises de dettes totalisant 1,1 milliard de francs, et des fonds de contrepartie pour un montant de 268 millions ont été institués dans 12 pays. Fin 1997, la Guinée a vu sa dette soulagée de 10,5 millions de francs et a créé un fonds de contrepartie de 1,05 million. De petites dettes yéménites ont été annulées en 1998. Des neuf pays restants, le Cameroun, Madagascar, la Sierra Leone et le Liberia pourraient bientôt bénéficier d'une opération de désendettement.

Jusqu'à l'été 1998, la Suisse avait versé 77 millions de francs à l'AID (filiale de la Banque mondiale) pour le rachat de dettes commerciales, ce qui en fait le premier pays donateur à titre bilatéral; 11 pays ont profité à ce jour des contributions helvétiques. Des dettes commerciales totalisant 1,8 milliard de dollars ont ainsi été rachetées et annulées avec les contributions d'autres donateurs. Les nouveaux bénéficiaires depuis le début de 1997 sont la Côte-d'Ivoire, la Guinée, la Guyane et la Mauritanie. Des négociations sont en cours pour le rachat de dettes commerciales de la Tanzanie.

Par contre, aucune opération de financement d'arriérés dus aux institutions financières internationales n'a eu lieu l'an passé. En 1997, la Suisse a pris en charge les échéances courantes de l'Ouganda et de la Bolivie à l'égard de ces institutions en prélude à l'initiative en faveur des PPTE. En 1998, elle a participé avec d'autres pays au financement de dettes de la Guinée-Bissau (avant la guerre) à l'égard des institutions multilatérales, et versé 8 millions de francs pour le Nicaragua. La Suisse entend à l'avenir faire passer ses apports dans ce domaine autant que possible par l'initiative PPTE. Elle appuiera également, dans ce contexte, des PPTE conformes aux critères de cette initiative mais qui n'en bénéficieront que plus tard.

Les mesures complémentaires comportaient notamment le versement d'argent frais sous forme d'aide à la balance des paiements de pays pauvres qui ont évité le surendettement grâce à une politique prudente. Plus aucune mesure de ce genre n'a été prise en 1997 et 1998. La Suisse propose par ailleurs une assistance technique en matière de désendettement. Elle continue d'une part de soutenir le programme d'informatisation de la gestion de la dette appliqué par la CNUCED; en 1997 et 1998, les pays bénéficiaires de contributions versées à cet effet par l'OFAEE ont été la Guinée-Bissau, la Bolivie, le Nicaragua, le Pérou, la Jordanie, le Vietnam et le Burkina-Faso. La Suisse est d'autre part l'initiatrice du programme de promotion de l'autonomie locale des PPTE, dont le but est d'aider les pays concernés à effectuer les analyses nécessaires dans le cadre de l'initiative PPTE. L'Autriche, le Danemark et la Suède participent à ce programme, et la Grande-Bretagne envisage de s'y joindre (voir plus haut la partie «Initiative en faveur des PPTE»). Ce programme sert en outre de plate-forme pour des échanges de vues réguliers entre acteurs qui s'occupent de gestion de la dette et de désendettement. Son comité de pilotage a réuni Banque mondiale, FMI, PNUD, CNUCED, PPTE et pays donateurs en juin 1998 à Berne, et en novembre de la même année à Londres. 
L'OFAEE suit de près d'autres sujets de préoccupation, tels que l'endettement à l'égard de nations non membres de l'OCDE et l'endettement interne de certains pays.

\subsection{INVESTISSEMENTS INTERNATIONAUX}

La CNUCED constate que les investissements directs internationaux ont atteint un nouveau record en 1997; les moteurs de cette évolution sont la libéralisation du commerce et des investissements ainsi que les privatisations. Les pays concernés souhaitent non seulement l'afflux de capitaux, mais aussi un meilleur accès à la technologie et au savoir. Les négociations relatives à un accord multilatéral sur l'investissement menées dans le cadre de l'OCDE ont pris fin sur un échec au début décembre 1998.

\section{$\square$ Rapport de la CNUCED sur l'investissement dans le monde}

Selon l'édition 1998 du rapport publié chaque année par la CNUCED, les investissements directs internationaux effectués dans le monde ont atteint quelque 3500 milliards de dollars, soit $13 \%$ de plus que 1'année précédente ${ }^{22}$. Un total de 2350 milliards de dollars étaient investis dans les nations industrialisées, et 1050 milliards dans les pays en développement et en transition.

Les nouveaux investissements directs effectués en 1997 se chiffrent à 400 milliards de dollars, dont 233 milliards ont pris le chemin de pays industrialisés, et 167 milliards celui de pays en développement ou en transition (tableau $\mathrm{n}^{\circ} 5$ ).

Tableau $n^{\circ} 5$ : Investissements directs étrangers par régions ou pays 1995-1997

\begin{tabular}{|c|c|c|c|c|c|c|}
\hline \multirow[b]{2}{*}{ Régions/Pays } & \multicolumn{3}{|c|}{ ID* entrants } & \multicolumn{3}{|c|}{ ID* sortants } \\
\hline & 1995 & 1996 & $1997^{p}$ & 1995 & 1996 & $1997^{\circ}$ \\
\hline Pays industrialisés & 211 & 195 & 233 & 306 & 283 & 359 \\
\hline Europe & 123 & 100 & 115 & 174 & 168 & 196 \\
\hline Amérique du Nord & 70 & 83 & 99 & 103 & 83 & 128 \\
\hline Japon & 0,0 & 0,2 & 3,2 & 23 & 23 & 26 \\
\hline Pays en développement & 106 & 130 & 149 & 46 & 49 & 61 \\
\hline Afrique & 5,1 & 4,8 & 4,7 & 0,5 & 0,3 & 1,1 \\
\hline Amérique latine / Caraïbes & 32 & 44 & 56 & 2,3 & 2,3 & 9,1 \\
\hline Asie & 67 & 80 & 87 & 42,6 & 46,5 & 50,6 \\
\hline dont Sud-Est asiatique & 67 & 77 & 82 & 41,8 & 47,3 & 50,2 \\
\hline Europe de l'Est & 14 & 12 & 18 & 0,5 & 1,0 & 3,3 \\
\hline Total & 331 & 338 & 400 & 353 & 334 & 424 \\
\hline
\end{tabular}

Source: CNUCED, Rapport sur l'investissement dans le monde 1998, tableau IB.1/B.2. $I^{*}=$ investissements directs

${ }^{\mathrm{p}}$ Chiffres provisoires.

L'essentiel de ces afflux reste limité à un nombre restreint de pays en développement, les principaux bénéficiaires du Sud et de l'Est étant la Chine (41 milliards de dollars), le Brésil (11), Singapour (9), le Mexique (8), l’Indonésie (6), l’Argentine (5), la Malaisie (5), la Pologne (5), le Chili (4) et le Pérou (4).

22. CNUCED, Rapport sur l'investissement dans le monde 1998. Tendances et déterminants, Nations Unies, New York et Genève, 1998. 
Le déséquilibre est encore plus frappant quand on considère l'origine de ces investissements directs, dont plus de $90 \%$ proviennent des pays industrialisés. Cependant, la part des pays en développement et en transition (donc près de $10 \%$ ) a plus que doublé depuis le début des années 90.

Les chiffres de la CNUCED ne reflètent pas encore la crise financière asiatique, mais cette institution a prévu que les investissements directs stagneraient au mieux en 1998 dans cette région du globe, tandis qu'ils devraient continuer d'augmenter en Amérique latine et dans les Caraíbes. Le rapport explique par ailleurs en détail pourquoi les investissements directs internationaux fluctuent beaucoup moins - sont moins volatiles - que les investissements de portefeuille.

La CNUCED constate dans son rapport une explosion des rachats d'entreprises par des sociétés multinationales, notamment lors de la privatisation d'entreprises publiques. Il en résulte également une concentration accrue de la propriété. Le rapport observe en outre un nombre croissant d'accords entre sociétés de pays différents, la plupart de ces contrats de coopération comportant d'ailleurs un volet technologique.

\section{$\square$ La Suisse et les investissements directs}

Le Rapport sur l'investissement dans le monde de la CNUCED indique que le montant des investissements directs étrangers en Suisse se chiffrait à 56,5 milliards de dollars en 1997, avec un afflux de 3,5 milliards presque identique à celui de l'année précédente. Inversement, les investissements directs suisses à l'étranger ont atteint 156,7 milliards de dollars, dont 12 milliards représentent l'accroissement de 1997. Le total des investissements directs suisses à l'étranger représente $49,2 \%$ du produit national brut, un taux qui place la Suisse loin devant tous les autres pays du monde.

La Banque nationale suisse estime que les investissements directs suisses dans des pays nouvellement industrialisés atteignaient en 1997 un volume de 726 millions de francs, contre 1406 millions l'année précédente ${ }^{23}$. Cette évolution s'explique partiellement par les retraits massifs d'investisseurs suisses au Brésil (-329 millions de francs). D'autre part, on ressent déjà les effets de la crise financière du Sud-Est asiatique; la Suisse a également désinvesti en Corée du Sud (-61 millions) et nettement ralenti le flux de ses investissements en Malaisie, à Singapour et en Thaillande (tableau $n^{\circ} 6$ ). Le total des investissements suisses dans les pays nouvellement industrialisés se chiffrait ainsi à 15’452 millions de francs à fin 1997.

On assiste par contre à un nouvel et important accroissement des investissements directs suisses dans les pays en développement, qui atteignent 1716 millions de francs contre 1057 millions l'année précédente, mais ils n'ont pas égalé le record de 2056 millions réalisé en 1991. Fin 1997, les capitaux suisses placés dans des pays en développement totalisaient 28'691 millions de francs, marquant ainsi une progression de $34 \%$ par rapport à l'année précédente.

Les investissements directs de pays nouvellement industrialisés en Suisse ont reculé en 1997 de 65 millions de francs pour tomber à 56 millions. Les pays en développement avaient à la même date pour 1292 millions de francs d'investissements directs en Suisse, soit 78 millions de plus que l'année précédente.

23. Banque nationale suisse, $4^{*}$ rapport trimestriel 1998, Zurich, décembre 1998. 
Tableau n6: Investissements directs de la Suisse dans des pays en développement et nouvellement industrialisés 1995-1997 (en millions de francs)

\begin{tabular}{lrrrrrr}
\hline & \multicolumn{3}{c}{ Stock des capitaux investis } & \multicolumn{2}{c}{ Flux d'investissements } \\
Région & $\mathbf{1 9 9 5}$ & $\mathbf{1 9 9 6}$ & $\mathbf{1 9 9 7}^{\mathrm{P}}$ & $\mathbf{1 9 9 5}$ & $\mathbf{1 9 9 6}$ & $\mathbf{1 9 9 7}^{\circ}$ \\
\hline Pays nouv. industrialisés & 11863 & 14044 & 15542 & 55 & 1406 & 716 \\
\hline Amérique latine & 7216 & 8528 & 8796 & 186 & 260 & -225 \\
\hline Asie & 4647 & 5516 & 6656 & -242 & 1146 & 951 \\
\hline Pays en développement & 19318 & 21393 & 28691 & 487 & 1057 & 1716 \\
\hline Amérique latine & 16524 & 17218 & 23329 & 2 & -267 & 839 \\
\hline Afrique & 1178 & 1290 & 1626 & 25 & 90 & 230 \\
\hline Asie & 1609 & 2886 & 3736 & 459 & 1234 & 648 \\
\hline
\end{tabular}

Source: Banque nationale suisse, $4^{e}$ rapport trimestriel 1998.

${ }^{\mathrm{p}}$ Chiffres provisoires.

\section{$\square$ Conventions d'investissement bilatérales}

Le nombre des accords bilatéraux en matière d'investissement a passé de 1330 à fin 1996 à plus de 1500 à fin 1997. La plupart définissent les modalités des investissements entre pays industrialisés et pays en développement ou en transition. Ces conventions représentent pour les premiers un moyen de protéger leurs investissements et d'en faciliter les aspects pratiques; pour les seconds, un moyen d'attirer des capitaux. On assiste également à une multiplication des accords d'envergure régionale. La CNUCED considère toutefois que ces ententes influent bien moins sur les décisions d'investir que d'autres facteurs - dimension du marché, prévisions de croissance, cours du change ou risques inhérents au pays considéré.

La CNUCED estime tout de même que des règles multilatérales seraient susceptibles de stimuler les investissements internationaux dans la mesure où elles procureraient une sécurité accrue aux investisseurs et davantage de stabilité, de prévisibilité et de transparence aux pays bénéficiaires.

\section{$\square$ Accord multilatéral sur les investissements (AMI)}

Faut-il considérer cet accord comme gelé sine die du fait que la France s'est retirée des négociations menées dans le cadre de l'OCDE? Ou bien l'OMC va-t-elle poursuivre le débat? Accordera-t-on une nouvelle chance à la CNUCED? Ou encore l'AMI ne verra-t-il jamais le jour?

Ces nombreux points d'interrogation reflètent la confusion qui règne autour de la question très controversée des règles multilatérales à appliquer en matière d'investissements. Il n'en reste pas moins que l'OCDE conduit des pourparlers à ce sujet depuis 1995, et a élaboré un projet d'accord.

Au départ, il y a l'absence de règles internationales applicables aux investissements, alors que les investissements internationaux croissent deux fois plus vite que le commerce mondial. Les règles nationales et régionales témoignent de tendances contradictoires. Un pays donné est constamment tenté d'instaurer des mesures protectionnistes afin de défavoriser les entreprises étrangères. D'autre part, le même pays, face à une concurrence géographique qui s'exacerbe, attire les investisseurs étrangers par des subventions de tout genre, souvent au mépris des normes sociales et écologiques en vigueur.

Les pays de l'OCDE ont entamé en 1995 des négociations censées déboucher sur une réglementation qui ne se limiterait pas aux membres de cette organisation, 
mais serait ouverte à tout pays qui souhaiterait y adhérer. Cependant, seuls quelques pays du Sud et de l'Est ont été invités en observateurs - et cela à un stade ultérieur des négociations concrètes.

Le principe fondamental de ce projet a toujours été la non-discrimination, c'està-dire un traitement égal des investisseurs indigènes et étrangers ainsi que l'application systématique de la clause de la nation la plus favorisée. L'accord prévu comprend aussi des règles portant sur le libre transfert de bénéfices et de capitaux, ainsi que sur des indemnisations équitables en cas d'expropriation. Il envisage des exceptions pour certains pays, de même que l'application de normes écologiques et sociales ${ }^{24}$. La procédure prévue pour l'arbitrage des différends privilégie les investisseurs.

Les négociations ont débuté à huis clos, mais la divulgation de leur teneur a aussitôt suscité, partout dans le monde, une résistance allant jusqu'au refus total. Ce projet d'accord a soulevé l'opposition d'organisations écologistes, culturelles et consuméristes aussi bien que des syndicats et des organisations tiers-mondistes. Les critiques portaient essentiellement sur le transfert de compétences gouvernementales à des investisseurs internationaux, ainsi que sur la procédure d'arbitrage international. Ce thème inédit a fait un tabac dans les médias. Les oppositions d'intérêts nationaux - en particulier entre les Etats-Unis et la France - sont devenues insurmontables, et la France s'est retirée des négociations en octobre 1998. Les pourparlers ont continué à l'échelon technique, pour être interrompus début décembre, sans résultat ${ }^{25}$.

La Suisse officielle était d'avis qu'une réglementation multilatérale servirait au mieux les intérêts helvétiques. L'OFAEE, notre organisme pilote en la matière, a même préconisé l'instauration de règles écologiques et sociales dans le cadre de cet accord. Les réserves critiques émises en Suisse sont venues de la Communauté de travail des œuvres d'entraide, de la Déclaration de Berne et des organisations écologistes ${ }^{26}$. De petites organisations plus intransigeantes - surtout genevoises - ont même appelé à la résistance totale. La question a donné lieu à plusieurs interventions parlementaires.

La position du Conseil fédéral peut se résumer comme suit: des règles multilatérales permettraient

- d'améliorer la sécurité du droit international;

- de juguler les excès de la concurrence géographique;

- de limiter le pouvoir des grands pays;

a d'instituer des normes sociales et environnementales.

Les organisations non gouvernementales, pour leur part, ont revendiqué entre autres l'inclusion des pays en développement dans les négociations, des restrictions aux investissements directs, des règles concernant la politique de concurrence, un équilibre entre les droits et les devoirs des investisseurs, etc.

24. Cf. OCDE, «L'AMI. L’Accord multilatéral sur l’investissement», Policy Brief, n² - 1997, Paris, 1997; Baldi, Marino, «L'Accord multilatéral sur l'investissement (AMI)», La Vie économique, 12/97, Berne, 1997.

25. «Abbruch der MAI-Verhandlungen», NZZ, 5.-6.12.98.

26. Gerster, Richard, «L'Accord multilatéral sur l'investissement (AMI) et le développement durable. La nécessité de garde-fous contraignants", La Vie économique, 1/98, Berne, 1998; Bosshard, Peter, et Sekinger, Urs, MAI - Freipass für Multinationale Konzerne?, Erklärung von Bern, Solifonds, Greenpeace, Zürich, April 1998. 


\subsection{INSTITUTIONS FINANCIÈRES INTERNATIONALES}

La crise financière plaçait le Fonds monétaire international (FMI) et la Banque mondiale devant un challenge inhabituel. Les deux institutions ont fait l'objet de critiques acerbes, ce qui a mis à rude épreuve leur collaboration et la répartition convenue de leurs activités. Aussi bien le FMI que la Banque mondiale ont accru leurs prêts dans des proportions considérables.

\subsubsection{LE FONDS MONÉTAIRE INTERNATIONAL}

Au cours de l'exercice 1997-1998 qui prenait fin le 30 avril 1998, le FMI, en raison de la crise financière, a sextuplé le volume de ses engagements par rapport à l'année précédente, et ses versements effectifs ont triplé dans le même laps de temps. Il en est résulté des problèmes de trésorerie, encore aggravés par le fait que les Etats-Unis tardaient à donner leur accord à l'augmentation de capital convenue. La ligne de conduite adoptée par le FMI durant la crise financière a provoqué de vives controverses.

\section{$\square$ Montant record des engagements}

La crise financière asiatique et russe $\mathrm{a}$ eu de très fortes répercussions sur les opérations financières du FMI, lequel a accordé des crédits qui totalisent 31,8 milliards de DTS (droits de tirage spéciaux) ou 42,8 milliards de dollars, soit six fois le montant de l'exercice précédent ${ }^{27}$. Les versements se sont chiffrés à 19,9 milliards de DTS, presque le quadruple de l'année précédente (tableau n ${ }^{\circ}$ ). Le FMI a conclu neuf accords relatifs à des crédits de disponibilité totalisant 27,3 milliards de DTS, dont 26,7 milliards avec des pays en crise du Sud-Est asiatique - Corée du Sud, Indonésie, Thaïlande et Philippines. Il a par ailleurs passé quatre accords mettant à contribution la Facilité élargie du fonds $(2,8$ milliards de DTS) et accordé huit nouveaux crédits (1,7 milliard) FASR (Facilité d'ajustement structurel renforcée). Fin avril, les accords en vigueur portaient sur 14 crédits de disponibilité, 13 crédits de la Facilité élargie et 33 crédits FASR. Les plus gros montants sont allés à la Corée (11,2 milliards de DTS), à l'Indonésie (2,2 milliards), à la Thaïlande ( 2 milliards) et à la Russie (1,5 milliard).

Tableau n7: Crédits du FMI 1995-1998 (en milliards de DTS, clôture au 30 avril)

\begin{tabular}{lrrrr}
\hline & $\mathbf{1 9 9 5}$ & $\mathbf{1 9 9 6}$ & $\mathbf{1 9 9 7}$ & $\mathbf{1 9 9 8}$ \\
\hline Versements & 11,2 & 12,3 & 5,6 & 19,9 \\
\hline$-\quad$ Afrique & 1,0 & 2,3 & 1,0 & 0,9 \\
\hline$-\quad$ Asie & 0,4 & 0,4 & 0,2 & 16,4 \\
\hline$-\quad$ Europe & 2,9 & 5,2 & 3,4 & 2,2 \\
\hline$-\quad$ Proche-Orient & 0,1 & 0,1 & 0,2 & 0,1 \\
\hline - Amérique latine & 6,8 & 4,4 & 0,9 & 0,3 \\
\hline Remboursements & 4,2 & 7,1 & 7,2 & 4,4 \\
\hline Transferts nets & 7,0 & 5,2 & $-1,6$ & 15,5 \\
\hline Crédits non remboursés & 36,8 & 42,0 & 40,5 & 56,0 \\
\hline Obligations non honorées à l'échéance & 3,0 & 2,2 & 2,2 & 2,3 \\
\hline Nombre de pays bénéficiares de crédits & 99,0 & 97,0 & 95,0 & 94,0 \\
\hline
\end{tabular}

Source: FMI, Rapport annuel 1998, tableaux 12 et 13.

27. FMI, Rapport annuel 1998, Washington, 1998. 
Le conseil d'administration a créé en décembre 1997 une facilité de réserve supplémentaire (Supplemental Reserve Facility) afin de pouvoir mettre rapidement de nouveaux fonds à la disposition des pays confrontés à des problèmes aigus de trésorerie. Ces ponctions considérables ont fait baisser le volume des fonds disponibles du FMI, qui sont ainsi tombés de 43,5 milliards de DTS l'année précédente à 22,6 milliards.

\section{$\square$ Renforcement des fonds propres}

La décision de principe prise l'année précédente d'augmenter les quotes-parts de $45 \%$ pour les porter à 212 milliards de DTS n'a pas pu se concrétiser durant l'exercice sous revue. Une coalition de députés conservateurs et écologistes a longtemps empêché le Congrès états-unien d'octroyer les ressources nécessaires. Il a fallu attendre la mi-octobre 1998 pour que la majorité républicaine du Congrès s'entende avec le président Clinton dans le cadre d'une enveloppe budgétaire globale, et donne ainsi son accord de principe à l'augmentation de capital du FMI. Une augmentation effective des quotes-parts requiert l'approbation de trois-cinquièmes des pays membres impliquant au moins $85 \%$ du capital, ce qui serait irréalisable sans l'apport des Etats-Unis.

Cette augmentation des quotes-parts fait régresser la part relative de la Suisse de 1,69 à $1,63 \%$ et celle du groupe de vote qu'elle conduit, de 2,7 à 2,6\%. Ce tassement relatif est dû à une adaptation des quotes-parts de plusieurs pays en fonction de leur importance réelle au sein de l'économie mondiale. Le Conseil fédéral a autorisé cet accroissement de la quote-part le 8 juin 1998. Dans une lettre adressée au conseiller fédéral Villiger, la Déclaration de Berne a accusé l'exécutif d'agir sans en informer d'abord le Parlement comme il l'aurait dû. La réponse du conseiller fédéral Villiger a rejeté ce reproche, et consécutivement à ce rejet le conseiller national Remo Gysin (PS, Bâle) a déposé une initiative parlementaire visant à soumettre les futures augmentations de capital du FMI à l'approbation du Parlement ${ }^{28}$.

Le Conseil des Etats a approuvé dès sa session d'automne 1997 l'adhésion de la Suisse aux Nouveaux Accords d'emprunt (NAE), et le Conseil national a fait chorus en décembre de la même année. Les NAE regroupent 25 pays, dont les 11 qui avaient déjà conclu les Accords généraux d'emprunt ( $A G E)$.

ASTM 1998, p. 159 sur les Nouveaux Accords généraux d'emprunt (NAE).

Les AGE ont été mis à contribution en juillet 1998 pour la Russie; ils ont servi à financer trois quarts du crédit alors accordé à ce pays. La Banque nationale suisse a versé une contribution de 378 millions de DTS (750 millions de francs).

\section{Surveillance}

Déjà renforcées l'année précédente, les activités de surveillance ont été encore intensifiées à la suite de la crise financière. Le FMI se réfère traditionnellement à l'article $I V$ «Consultation» pour examiner chaque année la politique économique des pays membres. S'il a accru sa surveillance en raison de la crise, le FMI

28. Bosshard, Peter, Alte Rezepte für neue Krisen? Die Rolle des Internationale Währungsfonds in Ostasien, die Kapitalerhöhung des IWF und die Schweiz, Erklärung von Bern, 10.8.98; lettre de la Déclaration de Berne du 4.6 .98 au conseiller fédéral Villiger; Initiative parlementaire Gysin Remo du 17.6.98: Approbation d'augmentations du capital du FMI par le Parlement. 
a également édicté des directives portant sur la bonne gestion des affaires publiques (good governance), le traitement des données économiques et la transparence en matière fiscale. Il a augmenté sa propre transparence en publiant désormais des rapports plus détaillés sur les consultations menées en vertu de l'article IV. Le FMI encourage ses membres à publier des lettres d'intention et des manifestes concernant leurs options politiques. Il a lui-même fait paraître ses rapports sur les PPTE et beaucoup d'autres documents jusque-là non accessibles au public.

En novembre de chaque année, une délégation du FMI vient examiner la politique économique et monétaire de la Suisse, conformément aux termes de l'article IV. Cette délégation a des entretiens approfondis avec les départements concernés de l'administration fédérale, la Banque nationale et les milieux économiques; elle rencontre également des représentants de la Communauté de travail des œuvres d'entraide. Celle-ci a soulevé en 1997 la question de la réforme fiscale écologique. En 1998, elle a demandé que la Suisse se montre plus active dans la lutte contre la crise financière; il faudrait prendre des mesures ciblées dans les pays les plus touchés pour venir en aide aux petites entreprises et tendre des filets de protection sociale. La Suisse devrait aussi favoriser les importations en provenance de ces pays.

\section{$\square$ Assistance technique}

Durant l'année écoulée le FMI a fourni de l'aide technique à hauteur de presque 300 hommes-années (postes à plein temps pendant une année). Ce poste représente $17 \%$ de ses dépenses administratives. L'assistance technique du FMI touche les domaines suivants (ordre d'importance décroissant): politique monétaire et problèmes de change, questions fiscales, statistiques, questions juridiques.

Le 15 avril 1998, la Suisse a conclu avec le FMI un accord concernant la création d'un fonds de 2,5 millions de dollars destiné à l'assistance technique du FMI aux pays d'Asie centrale faisant partie du groupe de vote qu'elle conduit. Cet argent a été versé par l'OFAEE ${ }^{29}$.

\section{$\square$ Facilité d'ajustement structurel renforcée}

L'instrument de prêts du FMI à taux d'intérêt préférentiels que constitue la Facilité d'ajustement structurel renforcée (FASR) a donné lieu à deux débats. D'une part, le financement intermédiaire décidé en 1996 n'est pas encore opératoire. Le Conseil devait présenter des rapports provisoires au Comité intérimaire pour la réunion de printemps aussi bien que pour la session annuelle de 1998. Les contributions bilatérales nécessaires ne sont toujours pas assurées. A fin juillet, seuls six pays avaient définitivement résolu de contribuer au fonds fiduciaire FASR-PPTE (voir plus haut la partie «Initiative en faveur des PPTE»). L'apport suisse, entériné en mars par le Conseil national, ne compte pas dans ces six pays.

Le Conseil a par ailleurs décidé en juin 1997 de soumettre la FASR à une évaluation approfondie par des experts internes et externes. Après des tergiversations initiales, le FMI a publié les deux rapports, qui ont donné lieu à des discussions nourries. Le rapport des experts internes ${ }^{30}$ plaide notamment pour une

29. IMF Survey, May 11, 1998, p. 145.

30. IMF, The ESAF at Ten Years. Economic Adjustment and Reform in Low-Income Countries, Occasional Paper 156, Washington DC, December 1997. 
amélioration des mesures d'adaptation fiscale, une lutte plus énergique contre l'inflation et des réformes structurelles plus profondes - restant ainsi enfermé dans le discours traditionnel du FMI. Les experts externes ont demandé une collaboration plus étroite avec la Banque mondiale dans le domaine social, en créant des liens entre macro- et microéconomie ainsi qu'avec l'analyse à court et à long terme. La question a été posée de savoir si la FASR devrait exercer une fonction d'appui après la phase de stabilisation. Les experts externes ont demandé que l'on prenne en considération des options politiques «alternatives» et que l'on fasse davantage pour l'émancipation (ownership) des pays considérés ${ }^{31}$.

\section{$\square$ Libéralisation des mouvements de capitaux}

Lors de la session annuelle tenue à Hongkong en automne 1997, le Comité intérimaire a décidé que le FMI aurait en principe pour tâche de promouvoir la libre circulation des capitaux. Il s'y tient en dépit de la crise financière, mais ses statuts n'ont pas encore été adaptés en conséquence.

Stanley Fisher, directeur général adjoint du FMI, a cependant relevé à plusieurs reprises qu'il serait très risqué de libéraliser les mouvements de capitaux sans que certaines conditions soient préalablement remplies - notamment une politique macroéconomique cohérente sans déséquilibre excessif de la balance des paiements et une surveillance appropriée du système financier. Il faut, selon Stanley Fisher, que le processus de libéralisation soit organisé de façon méthodique, avec une bonne coordination de chaque étape.

Beaucoup de critiques ont cependant demandé, après la crise financière, que l'on soumette les flux financiers - surtout ceux à court terme - à des contrôles et des restrictions.

\section{$\square$ Meilleure architecture du système financier international}

Dès après la crise financière du Mexique en 1994-1995, le FMI avait pris différentes initiatives pour renforcer le système financier international. En avril 1998, son conseil d'administration a répertorié une série d'autres leçons à tirer de la crise asiatique. Dès sa session de printemps, le Comité intérimaire a également donné son aval aux efforts prévus, qui englobent les mesures suivantes:

- renforcement des systèmes financiers international et locaux, amélioration des institutions de surveillance et de régulation, indépendance accrue des banques centrales, bonne gestion des affaires publiques, normes internationales de comportement;

- intensification de la surveillance exercée par le FMI, en particulier dans le secteur financier, collaboration étroite avec la Banque mondiale, la BRI et d'autres organisations internationales;

• transparence accrue, grâce à des données économiques de meilleure qualité et plus rapidement disponibles;

- confirmation du rôle moteur du FMI dans la gestion de la crise;

- association accrue du secteur privé dans le dépassement de la crise, par exemple en reportant des échéances et en procédant à des rééchelonnements de dettes. 
En avril 1998, les ministres des Finances et les gouverneurs des banques centrales des pays du G7, ainsi que 15 pays en développement et en transition économiquement importants de tous les continents, ont constitué ensemble trois groupes de travail (crise financière internationale; transparence et responsabilité; amélioration du système financier). La Suisse n'était pas de la partie et a protesté par écrit auprès des Etats-Unis, nation invitante. Elle a alors été conviée, avec les Pays-Bas, la Belgique et la Suède, à une séance d'information. Le FMI a publié les volumineux rapports des groupes de travail au début d'octobre sur Internet, en sollicitant des commentaires ${ }^{32}$.

\section{$\square$ Le FMI et la crise financière}

La crise financière a absorbé des ressources considérables (voir plus haut la partie «Montant record des engagements»), mais aussi occasionné énormément de travail aux responsables du FMI. Ceux-ci ont tenu de très nombreux exposés à ce sujet, qui a également fait l'objet d'articles dans une multitude de publications. La réponse politique apportée par le FMI peut se résumer comme suit:

- il faut garantir de manière crédible aux investisseurs autochtones et étrangers que l'équilibre macroéconomique sera rétabli;

- des mesures fiscales sont nécessaires, mais ne doivent pas trop compromettre la demande globale; il faut en particulier amortir les coûts sociaux et assurer la restructuration du système financier;

- la politique monétaire doit tenir son cap, pour éviter les dévaluations lourdement inflationnistes et éviter la fuite de capitaux;

- il faut combler le plus vite possible les déficits structurels qui peuvent affecter le secteur financier de certains pays;

- la bonne gestion des affaires publiques doit être garantie.

Ce qui a été le plus critiqué dans cette politique, ce sont les restrictions budgétaires et les taux d'intérêt élevés qui ont trop freiné la demande et entraîné une grave récession dans les pays concernés ${ }^{33}$.

Dans l'intervalle, le FMI a autorisé des déficits budgétaires plus importants dans ces pays et moins de rigueur dans la rémunération des capitaux (voir plus haut la partie «La crise financière»).

\subsubsection{LA BANQUE MONDIALE}

\section{$\square$ Activités au cours de l'exercice}

La Banque mondiale a accordé durant l'exercice écoulé (1.7.97-30.6.98) les plus gros crédits jamais octroyés depuis sa création, à savoir 21,1 milliards de dollars pour 151 projets par la BIRD (Banque internationale pour la reconstruction et le développement), et 7,5 milliards pour 135 projets par l'AID. Cela s'explique par la crise financière et par des besoins accrus, de pays africains surtout.

Les versements de la Banque ont totalisé 25,5 milliards de dollars, soit 19,8 milliards par la BIRD et 5,6 milliards par l'AID (tableau n8).

Le bénéfice net de 1,234 milliard de dollars se situe au même niveau que l'année précédente, mais on s'attend pour l'avenir à ce que les gains diminuent peu à peu.

32. IMF, Reports on the International Financial Architecture, Washington, 1998.

33. Cf. «Globale Entwicklungsfinanzierung», Mosquito, Nr. 5, August 1998. 
Cela devrait provoquer des conflits d'intérêts entre trois catégories de pays: les nations industrialisées, celles qui se caractérisent par des revenus moyens, et les plus pauvres. Les pays industrialisés auront tendance à vouloir diminuer leurs propres contributions et à faire un usage accru des bénéfices nets (par exemple pour augmenter le capital de l'AMGI - Agence multilatérale de garantie des investissements). Les pays à revenus moyens voudraient payer moins d'intérêts sur leurs crédits, ce qui réduirait le bénéfice de la Banque mondiale. Et les pays pauvres souhaiteraient que ce bénéfice serve à financer des tâches spéciales (par exemple l'initiative en faveur des PPTE).

Tableau n`8: Flux de ressources de la Banque mondiale 1995-1998 (en milliards de dollars, clôture au 30 juin)

\begin{tabular}{lrrrr}
\hline & $\mathbf{1 9 9 5}$ & $\mathbf{1 9 9 6}$ & $\mathbf{1 9 9 7}$ & $\mathbf{1 9 9 8}$ \\
\hline Banque mondiale & & & & \\
\hline Engagements & 16,9 & 14,7 & 13,4 & 21,1 \\
\hline Versements & 12,7 & 13,4 & 14,0 & 19,2 \\
\hline Versements nets & 0,9 & 1,2 & 2,1 & \\
\hline Crédits non utilisés & 123,5 & 164,8 & 157,4 & 157,6 \\
\hline AID & & & & \\
\hline Engagements & 5,7 & 6,9 & 4,6 & 7,5 \\
\hline Versements & 5,7 & 5,9 & 6,0 & 5,6 \\
\hline Versements nets & 5,2 & 5,3 & 5,2 & 4,8 \\
\hline
\end{tabular}

Source: Banque mondiale, Rapport annuel 1998, Washington, 1998.

Le plus gros emprunteur a été la République de Corée avec un montant record de 3 milliards de dollars, suivie par la Chine, l'Inde, le Mexique, la Russie, le Brésil et l'Argentine, qui ont tous obtenu des crédits dépassant le milliard de dollars.

Les versements de la Banque mondiale se répartissent géographiquement de la manière suivante (en milliards de dollars):

$\begin{array}{llll}\text { Asie du Sud-Est } & 9,62 & \text { Asie du Sud } & 3,86 \\ \text { Amérique latine (Caraibes) } & 6,04 & \text { Afrique } & 2,87 \\ \text { Europe/Asie centrale } & 5,22 & \text { Proche-Orient/Afrique du Nord } & 0,97\end{array}$

Dans l'octroi de ses crédits la Banque mondiale a donné la priorité aux secteurs éducatif, sanitaire, alimentaire, démographique et social. Les montants affectés à ces domaines ont totalisé 6,4 milliards de dollars, et même 8,5 milliards si l'on y inclut la composante sociale d'autres prêts, soit $30 \%$ de tous les crédits accordés par la Banque.

\section{$\square$ La Banque mondiale et la crise financière}

Au cours de cette crise, la Banque mondiale a essentiellement aidé ses membres à en surmonter les répercussions sociales. Elle considère que les pays touchés doivent pouvoir maintenir et améliorer leurs services sociaux, qu'il convient donc de mettre en place et de financer des fonds sociaux et de renforcer les systèmes de protection sociale.

Mais la Banque mondiale appuie aussi les réformes du secteur financier. Elle vise indirectement à rétablir la croissance par des réformes de la politique en matière de concurrence et par une efficacité accrue des institutions publiques - ce qui inclut la lutte contre la corruption. 
La Banque mondiale a octroyé aux pays touchés par la crise financière un total de 16 milliards de dollars, dont 5,65 milliards avaient été versés jusqu'à juin 1998.

Etant donné les problèmes de trésorerie qui affectaient le FMI, la Banque mondiale s'est vue très sollicitée d'apporter elle-même de l'aide dans ce domaine. Mais son président, James Wolfensohn, a clairement fait comprendre que les tâches incombant à la Banque mondiale lui interdisaient de procéder à des injections de liquidités. Si elle devait mettre davantage d'argent à disposition, il faudrait augmenter son capital.

La direction de la Banque mondiale a par ailleurs émis des avis divergents par rapport au FMI. Lors de son allocution à la session annuelle, le président Wolfensohn a souligné qu'il ne suffirait pas de stabiliser les systèmes financiers pour résoudre une crise dans ce domaine. Politique financière et politique sociale devraient aller de pair. Joseph Stiglitz, économiste en chef de la Banque mondiale, s'est exprimé encore plus clairement à différentes occasions. Des agences ont rapporté notamment que lors d'un exposé à la CNUCED à Genève, il a soutenu que le «consensus de Washington» (ligne politique convenue entre les principaux acteurs de l'économie mondiale) était dépassé et devait être réformé.

\section{$\square$ Rénovation de l'institution (le Pacte stratégique)}

@ ASTM 1998, pp. 162-163 sur le Pacte stratégique.

Cette rénovation a pour but de rendre la Banque mondiale plus apte à remplir sa fonction essentielle, qui est de combattre la pauvreté. Son Rapport annuel 1998 montre que la qualité des projets présentés s'est encore améliorée, que la proportion de projets entravés par des difficultés diminue et qu'il faut encore moins de temps pour préparer de nouveaux projets - alors même que leur nombre augmente et implique globalement un montant plus élevé.

La Banque mondiale a en outre élaboré de nouveaux instruments; deux d'entre eux concernent les garanties. L'AID peut désormais garantir des crédits privés, tandis que la BIRD a la possibilité de prendre en charge certains risques de créanciers privés dans des pays où l'AID est par ailleurs seule à opérer. De plus, la Banque a instauré des crédits de formation et d'innovation atteignant 5 millions de dollars au maximum, que sa direction est habilitée à octroyer de sa propre compétence.

Les quatre réseaux thématiques institués l'année précédente (développement humain, HD; développement écologiquement et socialement durable, ESSD; finances, développement du secteur privé et infrastructure, FPSI; lutte contre la pauvreté et gestion économique, PREM) font un travail qui, au dire de la Banque, accroît son aptitude à transmettre des connaissances et à apprendre elle-même.

Un comité spécial du conseil d'administration, le CODE, s'occupe plus particulièrement d'améliorer l'efficacité de l'institution et d'examiner dans quelle mesure la direction met en œuvre les recommandations de l'instance d'évaluation interne ( $O E D$ - Département de l'évaluation des opérations).

Le conseil d'administration a d'autre part promulgué des directives et des stratégies concernant la bonne gestion des affaires publiques et la lutte contre la corruption.

Le Panel d'inspection reste passablement controversé. Cet organe de médiation de la Banque mondiale est vivement critiqué par de nombreux gouvernements de 
pays en développement. Ceux-ci apprécient peu qu'un organe indépendant contrôle si l'on respecte les dispositions juridiques de la Banque mondiale lors de la réalisation des projets, car cela peut mettre en lumière leurs propres irrégularités. La plupart des pays industrialisés - Suisse comprise - sont au contraire plutôt disposés à accorder au Panel d'inspection une certaine autonomie dans l'instruction de plaintes contre la Banque mondiale.

\section{$\square$ Autres éléments importants du programme}

La pauvreté ne peut se combattre qu'avec la participation active des populations directement concernées. La Banque mondiale constitue pour ce faire des groupes de partenariat incluant d'autres institutions, l'économie privée et le secteur civil. Elle coopère de plus en plus avec des organisations non gouvernementales, des syndicats et des groupes locaux pour l'élaboration de ses stratégies d'aide aux pays (SAP). La DDC participe à un programme de contrôle de ces SAP dans certains pays.

Le Rapport sur le développement dans le monde 2000 reviendra, après celui de 1990, sur les stratégies adoptées pour réduire la pauvreté. Des organisations non gouvernementales sont associées aux travaux préparatoires.

Dans le domaine des microcrédits aux pauvres, 25 pays - dont la Suisse - ont adhéré au Groupe consultatif pour aider les plus pauvres (CGAP). Ce groupe a financé jusqu'à présent 58 programmes innovateurs.

La Banque mondiale a poursuivi sa politique d'égalité entre hommes et fermmes. Les éléments relevant de cette préoccupation ont totalisé 2451 millions de dollars au cours de l'exercice écoulé. Bien que la Banque déclare améliorer constamment cet aspect dans ses analyses, projets, programmes et textes juridiques, elle reste la cible de critiques virulentes.

Les esprits se sont un peu calmés en ce qui concerne la politique environnementale de la Banque mondiale, dont la revue Environment Matter ${ }^{34}$ donne un aperçu. Après avoir régulièrement augmenté ces dernières années, le montant globalement affecté à des projets environnementaux a de nouveau reculé en 1998; il se chiffre à 10'930 millions de dollars contre 11'600 millions pour l'exercice précédent. La Banque remanie actuellement ses documents stratégiques concernant l'énergie et la foresterie.

\section{$\square 11^{e}$ et $12^{e}$ reconstitution du fonds de l'AID}

L'Association internationale de développement (AID) est réalimentée tous les trois ans. Ses crédits s'étendent sur trente-cinq à quarante ans avec un différé d'amortissement de dix ans, et sont octroyés sans intérêt. La $11^{\mathrm{e}}$ reconstitution du fonds de l'AID a été financée par 40 pays, dont la Corée, la Turquie et le Botswana, qui en obtiennent également des crédits. Il y a aujourd'hui 80 pays habilités à obtenir des prêts de l'AID; leurs habitants sont au nombre de 3,3 milliards et représentent $80 \%$ de la population des pays en développement. Et 1,3 milliard d'entre ces personnes survivent avec moins d'un dollar par jour. Les Etats-Unis sont toujours en retard dans le versement de leurs contributions. Cependant, le

34. The World Bank, Environment Matter: Annual Review, Fall 1998, Environment and Socially Sustainable Development Network, Washington, 1998. 
nouvel arrangement budgétaire entre le gouvernement Clinton et la majorité républicaine du Congrès devrait aboutir au règlement de ces arriérés.

Les pourparlers relatifs à la $12^{\mathrm{e}}$ reconstitution du fonds ont débuté en février 1998 à Paris; après d'autres rencontres en mai à Londres et durant la session annuelle à Washington, ces négociations ont trouvé leur conclusion en novembre à Copenhague. Au cours de cette $12^{\mathrm{e}}$ période de trois ans qui débutera le $1^{\text {er }}$ juillet 1999 , l'AID disposera, pour le financement de projets, d'une enveloppe de 20,5 milliards de dollars dont 11,6 milliards proviennent de nouvelles contributions des pays donateurs. Le reste provient essentiellement des remboursements d'anciens prêts de l'AID et des bénéfices de la Banque mondiale ${ }^{35}$. Il y avait 22 milliards de dollars à disposition pour la $11^{\mathrm{e}}$ période.

L'apport de la Suisse est de 2,43\% ou 420 millions de francs en tout. Les contributions annuelles devront s'inscrire dans le cadre du budget normal.

\section{$\square$ La promotion du secteur privé}

Le renforcement du programme «Finances, développement du secteur privé et infrastructure» s'est poursuivi. Les fonds affectés à ce programme ont augmenté de deux tiers par rapport à l'année précédente, surtout en raison des mesures entraînées par la crise financière et d'une demande croissante des secteurs énergétique, minier et industriel.

La Banque mondiale entend mobiliser des capitaux privés par le biais de garanties pour les crédits et les investissements. Elle encourage également le développement du secteur privé afin de soutenir la création d'emplois, et a fondé l'an dernier l'institution Business Partners for Development. Le programme de petits crédits du CGAP fait également partie de ce département de la Banque.

La Société financière internationale (SFI) a investi durant l'exercice écoulé dans 226 projets un total de 5,1 milliards de dollars, sous forme de crédits ou de participations ${ }^{36}$. Sa priorité au cours de cet exercice a été de renforcer les marchés financiers locaux. Après quelques hésitations, la SFI s'est dotée de principes politiques plus rigoureux dans les domaines écologique et social ainsi qu'en matière d'information des milieux concernés.

Les activités de l'Agence multilatérale de garantie des investissements (AMGI) se sont beaucoup intensifiées au cours de l'exercice 1998, passant de 614 millions de dollars l'année précédente à 830,9 millions, dans 26 pays $^{37}$. Dans dix d'entre eux - dont l'Angola, la Bolivie, le Cap-Vert et le Mozambique - c'était la première fois qu'elle apportait sa garantie. Les projets AMGI devraient avoir contribué à créer quelque 8000 emplois et attiré plus de 6 milliards de dollars en investissements directs dans ces pays. Selon l'évaluation effectuée durant l'exercice sous revue, les investissements appuyés par l'AMGI ont eu des effets positifs sur le développement économique des pays concernés ${ }^{38}$.

35. The World Bank, Poorest Countries to Receive More than $\$ 20$ Billion from IDA, The World Bank News Release, Nr 99/2011/S, November 19, 1998.

36. SFI, Rapport annuel 1998, Washington, 1998

37. AMGI, Rapport annuel 1998, Washington, 1998.

38. West, Gerald T. and Tarazona, Ethel I., MIGA and Foreign Direct Investment: Evaluating Developing Impacts, MIGA, Washington, 1998. 
Le rapport de cette année est consacré au thème du savoir. Il analyse les risques et le potentiel de la «révolution globale de l'information» pour les pays en développement. Il constate que l'accès au savoir financier, technique et médical est crucial pour améliorer le niveau de vie dans ces pays. James Wolfensohn fait remarquer dans sa préface que les nouvelles technologies éliminent d'une part des obstacles géographiques et temporels, mais qu'elles risquent d'autre part d'accroître le retard des pays pauvres.

Ce rapport a été présenté en Suisse lors d'un atelier organisé par la DDC et l'OFAEE en présence d'experts de la Banque mondiale.

\subsubsection{SESSION ANNUELLE DU FMI ET DE LA BANQUE MONDIALE}

Le thème central de cette session a été, bien entendu, la crise financière. Les nombreux entretiens entre ministres des Finances, gouverneurs de banques centrales, responsables du FMI et de la Banque mondiale, banquiers et ONG ont mis en évidence des opinions divergentes sur la manière de sortir le monde de cette crise. Beaucoup de gouvernements ont appelé le secteur privé à se montrer plus coopératif et à retirer moins vite et de façon moins draconienne ses capitaux des régions en crise. Le secteur privé, de son côté, a demandé aux gouvernements et aux institutions financières internationales de commencer par rétablir la confiance au moyen d'une politique appropriée. Les gouvernements européens ont critiqué la domination des Etats-Unis dans les institutions de Bretton Woods. Les nouveaux gouvernements sociaux-démocrates d'Europe se sont montrés assez disposés à accepter certains contrôles des marchés financiers. Le président des Etats-Unis, de son côté, a demandé la création d'une nouvelle facilité de crise qui devrait permettre au FMI d'octroyer rapidement de l'aide financière aux pays qui rencontrent des difficultés en dépit d'une politique économique valable; ce serait un moyen de freiner la propagation de la crise financière.

Cette crise financière a fait passer les questions écologiques et sociales au second plan. Mais le thème de la transparence était omniprésent, alors même que cette exigence s'adresse bien plus aux pays qui obtiennent des crédits qu'à ceux qui les octroient.

\section{$\square$ Comité intérimaire}

Carlo Azeglio Ciampi, ministre italien des Finances, a été nommé nouveau président du Comité intérimaire. Celui-ci a donné son aval à la politique pratiquée jusque-là par le FMI, tout en appelant les pays industrialisés à assumer la responsabilité qui leur incombe de promouvoir une croissance durable de l'économie mondiale. Au lieu de combattre avant tout les risques d'inflation, il faudrait créer les impulsions nécessaires à une croissance accrue. Le Comité intérimaire se réjouit des propositions émises en vue d'une nouvelle architecture du système financier international et souhaite un renforcement de la collaboration entre FMI et Banque mondiale.

\section{$\square$ Comité de développement}

Ce comité s'est occupé lui aussi des problèmes posés par la crise asiatique, la collaboration avec le FMI et les défis que cela représente pour la Banque mon- 
diale. Son communiqué loue à mots couverts les efforts de la Banque pour aider les pays concernés à réformer les structures de leur secteur financier, à atténuer les répercussions sociales de la crise et à créer un climat favorable à la reprise économique.

\section{$\square$ Session annuelle}

Parmi les nombreux discours tenus comme de coutume devant l'assemblée plénière, celui de James Wolfensohn, président de la Banque mondiale, a été particulièrement remarqué. Son affirmation centrale est qu'il ne suffit pas aujourd'hui de résoudre la crise financière à court terme et de mettre en place une nouvelle architecture dans ce domaine: «Si nous ne sommes pas capables de nous intéresser à la détresse sociale, si nous n'avons pas de plans à long terme pour des institutions solides et si nous n'arrivons pas à créer davantage d'égalité et de justice sociale, il n'y aura pas de stabilité politique. Et sans stabilité politique, les plus gros crédits ne parviendront pas à garantir la stabilité financière.»

Le conseiller fédéral Kaspar Villiger, ministre des Finances, a présenté cinq propositions pour une stratégie. Transparence et association du secteur privé sont des postulats déjà évoqués plus haut. Le représentant de la Suisse a en outre appelé le FMI et la Banque mondiale à accomplir leurs missions initiales. Avant de créer de nouveaux instruments et d'engager de nouveaux capitaux, il convient d'exploiter jusqu'au bout ceux dont on dispose. Mais il faudrait en premier lieu que chaque pays mette de l'ordre dans sa propre maison. Seuls devraient bénéficier d'une aide financière les pays qui poursuivent une politique valable. 


\section{SOURCES}

Banque des règlements internationaux (BRI):

BRI, $68^{\circ}$ rapport annuel, Bâle, juin 1998.

BRI, Activité bancaire et financière internationale, Bâle, août 1998.

\section{Institutions de Bretton Woods:}

Fonds monétaire international (FMI-IMF)

FMI, Rapport annuel 1998, Washington, 1998.

IMF, Official Financing for Developing Countries, World Economic and Financial Surveys, Washington, February 1988. IMF, Debt Initiative for the Heavily Indebted Poor Countries, Fact Sheet, http://www.imf/org/external/np/hipc/Hipc/htm, September 1998.

IMF, HIPC Initiative: A Progress Report, Press Release, September 25, 1998.

IMF, The ESAF at Ten Years: Economic Adjustment and Reform in Low-Income Countries, Occasional Paper 156, Washington DC, December 1997.

IMF, External Evaluation of the ESAF: Report by a Group of Independent Experts, Washington DC, June 24, 1998.

IMF, Reports on the International Financial Architecture, Washington, 1998.

$I M F$ Survey, différents numéros.

Banque mondiale (BM-WB)

BM, Rapport annuel 1998, Washington, 1998.

WB, Global Development Finance 1998, 2 vol., Washington, 1998.

WB, HIPC Debt Initiative: Taking Stock and Looking Ahead, Conference Documents, The World Bank, February 3 , 1998, Paris.

SFI, Rapport annuel 1998, Washington, 1998.

AMGI, Rapport annuel 1998, Washington, 1998.

West, Gerald T. and Tarazona, Ethel I., MIGA and Foreign Direct Investment: Evaluating Developing Impact, MIGA, Washington, 1998.

WB, Poorest Countries to Receive More than \$20 Billion from IDA, The World Bank News Release, Nr. 99/2011/S, November 19, 1998.

WB, Environment Matters. Annual Review, Environment and Socially Sustainable Development Network, Washington, 1998.

OCDE:

OCDE, L'aide et les apports de capitaux privés ont diminué en 1997, communiqué de presse, Paris, 18 juin 1998.

OCDE, Coopération pour le développement. Efforts et politiques des membres du Comité d'aide au développement. Rapport 1997, Paris, 1998

OCDE, «L'AMI. L'Accord multilatéral sur l'investissement», Policy Brief, n² - 1997, OCDE, Paris, 1997.

$O N U$ :

CNUCED, Rapport sur l'investissement dans le monde 1998. Tendances et déterminants, Nations Unies, New York et Genève, 1998.

Autres:

«Rapport sur la politique économique extérieure 1997» du Conseil fédéral, Feuille fédérale, 10.3.98.

Baldi, Marino, «L'Accord multilatéral sur l'investissement (AMI)», La Vie économique, 12/97, Berne, 1997.

Banque nationale suisse, 4 e rapport trimestriel 1998, Zurich, décembre 1998.

Bosshard, Peter, Alte Rezepte für neue Krisen? Die Rolle des Internationalen Währungsfonds in Ostasien, die Kapitalerhöhung des IWF und die Schweiz, Erklärung von Bern, 10.8.98.

Bosshard, Peter, et Sekinger, Urs, MAI - Freipass für Multinationale Konzerne ?, Erklärung von Bern, Solifonds, Greenpeace, Zürich, April 1998.

Gerster, Richard, «L'Accord multilatéral sur l'investissement (AMI) et le développement durable. La nécessité de gardefous contraignants», La Vie économique, 1/98, Berne, 1998.

GRE/OFAEE, Rapport GRE 97, Bureau GRE/OFAEE, Zurich/Berne, juin 1998.

Info@Bawi, n²/1998 (e-mail info@bawi.admin.ch).

Initiative parlementaire Gysin Remo du 17.6.98: Approbation d'augmentations du capital du FMI par le Parlement.

Financial Times, «The Banks in East Asia», 10.6.98.

Mosquito, «Globale Entwicklungsfinanzierung», Nr. 5, August 1998.

Neue ZürcherZeitung, «Abrupt versiegende Kapitalströme im Jahr 1998», 30.1.98; «Die Weltwirtschaft im Banne der Asien-Krise», 9.6.98; «Abbruch der MAI-Verhandlungen», 5.-6.12.98. 